\title{
Clinical trials registries are under-utilized in the conduct of systematic reviews: a cross-sectional analysis
}

\author{
Christopher W Jones ${ }^{1 *}$, Lukas G Keil ${ }^{2}$, Mark A Weaver ${ }^{3}$ and Timothy F Platts-Mills ${ }^{2}$
}

\begin{abstract}
Background: Publication bias is a major threat to the validity of systematic reviews. Searches of clinical trials registries can help to identify unpublished trials, though little is known about how often these resources are utilized. We assessed the usage and results of registry searches reported in systematic reviews published in major general medical journals.

Methods: This cross-sectional analysis includes data from systematic reviews assessing medical interventions which were published in one of six major general medical journals between July 2012 and June 2013. Two authors independently examined each published systematic review and all available supplementary materials to determine whether at least one clinical trials registry was searched.

Results: Of the 117 included systematic reviews, 41 (35\%) reported searching a trials registry. Of the 29 reviews which also provided detailed registry search results, 15 (52\%) identified at least one completed trial and 18 (62\%) identified at least one ongoing trial.

Conclusions: Clinical trials registry searches are not routinely included in systematic reviews published in major medical journals. Routine examination of registry databases may allow a more accurate characterization of publication and outcome reporting biases and improve the validity of estimated effects of medical treatments.
\end{abstract}

Keywords: ClinicalTrials.gov, Systematic review, Trials registry, Publication bias

\section{Background}

Systematic reviews are an important means of synthesizing medical research findings to inform medical decision making. The validity of conclusions from systematic reviews depends on the authors' abilities to identify all previously conducted research relevant to the subject and of sufficient quality to be informative. Because non-publication of original research results that are negative or undesirable threatens the validity of conclusions from systematic reviews, methods have been developed to assess the identified literature for publication bias. These methods, which include the inspection and testing of funnel plots, are now routinely performed and explicitly mentioned in the guidelines for the reporting of systematic reviews [1-3]. However, these methods are

\footnotetext{
* Correspondence: Cjones.unc@gmail.com

${ }^{1}$ Department of Emergency Medicine, Cooper Medical School of Rowan University, One Cooper Plaza, Suite 152, Camden, NJ 08103, USA Full list of author information is available at the end of the article
}

imperfect. At best they suggest the presence of unpublished small- or moderately-sized studies based on the assumption that the largest studies on the subject are published, an assumption which may often be incorrect [4]. Empirical analyses indicate that these methods do not reliably detect publication bias [5,6].

Clinical trials registries allow researchers, clinicians, and the general public to learn about clinical trials conducted on a subject regardless of whether the results have been published. Since 2005, the International Committee of Medical Journal Editors (ICMJE) has required that prospective trials involving human participants undergo registration prior to initiating study enrollment as a condition of publication in member journals [7]. Following the passage of the United States Food and Drug Administration Amendments Act (FDAAA) in 2007, prospective trial registration became a requirement under United States law for many interventional clinical trials [8]. Thus, clinical trials 
registries now form a more comprehensive repository of recently initiated clinical trials. Policies supporting trial registration have been motivated in large part by the belief that consistent pre-registration of clinical trials will help consumers of the medical literature monitor and account for publication bias and other forms of selective reporting $[7,9,10]$.

Awareness of clinical trial results which remain unpublished and unavailable can have important consequences for the conclusions drawn from systematic reviews [11]. Commonly referenced guidelines for the conduct of systematic reviews recommend a search of trials registries as part of a comprehensive search strategy $[12,13]$. However, whether authors of systematic reviews typically comply with these guidelines is unclear. Our objective was to determine what proportion of authors of systematic reviews recently published in high-impact general medical journals searched clinical trials registries in order to identify relevant unpublished studies.

\section{Methods}

\section{Data sources}

We identified systematic reviews published between July 1, 2012 and June 30, 2013 in six high-impact general medical journals: Annals of Internal Medicine, BMJ, The Journal of the American Medical Association (JAMA), The Lancet, The New England Journal of Medicine, and PLoS Medicine. These journals were chosen because the manuscripts contained therein have a substantial influence on clinical practice and because they also set precedent for medical research methodology. Systematic reviews were identified by searching MEDLINE via PubMed using the following search terms: (Meta-analysis[Publication Type] OR meta-analysis[Title/Abstract] OR meta-analysis[MeSH Terms] OR review[Publication Type] OR search*[Title/ Abstract]). This search strategy has been shown to be sensitive for the identification of published systematic reviews [14]. We used the advanced search feature of PubMed to restrict the search to manuscripts with publication dates between July 1, 2012 and June 30, 2013 in the six aforementioned journals.

One study author (CWJ) reviewed the full manuscripts identified during this initial search to determine whether or not each publication included a systematic review. We classified articles as systematic reviews based on previously developed criteria: the manuscript included a clear statement identifying the topic of the review; authors provided a detailed description of the methods and datasources used to identify evidence included in the review; the manuscript included explicit inclusion and exclusion criteria; and the results included at least one study which met these inclusion criteria [14]. Two study authors (CWJ and LGK) evaluated the included systematic reviews to determine whether the primary goal of each was to assess the effects of an intervention.

\section{Outcome measures}

For each systematic review, two authors (CWJ and LGK) independently reviewed the full text, including appendices and online supplements. The primary outcome was whether the systematic review included a search of at least one clinical trials registry. ClinicalTrials.gov and those trials registries meeting World Health Organization (WHO) Primary Registry Criteria, version 2.1, were included [15] The WHO criteria require that registries pledge to make prospectively registered trial information freely available to the general public. Additional requirements include the ability to track and record any changes made to registry entries, implementation of quality control procedures to ensure the accuracy and completeness of registry data, and management by a not-for-profit agency. Fourteen registries met these requirements at the time of our analysis, including the International Standard Randomised Controlled Trial Number Register and the European Union Clinical Trials Register [15]. Discrepancies between authors with respect to trial registry utilization among the included systematic reviews were resolved by consensus.

\section{Study variables}

When authors indicated that a trials registry search was part of their review protocol, we reviewed the manuscript and all available supplementary materials to determine the number of relevant ongoing or completed studies which were identified as a result of the registry search. For the reviews in which this information was ambiguous or unavailable we emailed the corresponding author to request additional details regarding their search results. We also reviewed full manuscripts and all available supplementary materials to determine whether review authors provided search terms which would allow for the replication of the registry search. We searched each manuscript for a statement from study authors indicating whether the review was compliant with the Preferred Reporting Items for Systematic Reviews and Meta-Analyses (PRISMA) reporting guidelines for systematic reviews. Additionally, we gathered information about study funding and author financial disclosures for each manuscript. Both author support and direct study funding were considered together when categorizing reviews by funding source.

Because clinical trials are more commonly registered than observational studies, registry data are likely to be most relevant to those systematic reviews which evaluate interventions. For this reason, our analysis was limited to those systematic reviews which had a stated primary goal of assessing an intervention in a human population. 


\section{Statistical analysis}

Inter-rater agreement for our primary outcome was calculated using Cohen's kappa. Statistical analyses were performed using SPSS version 20.0 (IBM, Armonk, New York).

\section{Results}

Our PubMed search retrieved 567 records published between July 1, 2012 and June 30, 2013, 177 of which were determined to be systematic reviews (Additional file 1). Of these, 117 (66\%) had a stated primary objective of assessing the effects of an intervention and therefore compose our primary study sample. A search of one or more clinical trials registries was described as part of the literature search protocol in 41 (35\%) of these 117 manuscripts (Table 1). Subgroup analysis by journal and by funding source showed that there was no subgroup in this sample for which searches of clinical trial registries occurred in more than $50 \%$ of the systematic reviews. For 37 reviews, authors reported searching ClinicalTrials.gov either directly or through a registry search portal (International Clinical Trials Registry Platform (ICTRP) or Current Controlled Trials). Nineteen of these 37 reviews included searches of other registries in addition to ClinicalTrials. gov. Four reviews did not specify which trials registries were searched. For the remaining 76 reviews (65\%), authors did not report searching a registry.

Detailed registry search results were available for 29 reviews. Authors reported finding at least one relevant completed study as a result of their registry search for 15 of these 29 reviews (52\%) and at least one ongoing study for 18 of these reviews (62\%; Figure 1). Twenty-three of the 29 reviews (79\%) found at least one completed or ongoing study during the registry search. The authors of one of these 23 reviews indicated that they had identified both relevant completed and ongoing studies as a result of their registry search, but they were unable to provide the specific number of studies in each category [16]. Among the reviews for which specific data were available, the median number of relevant studies identified through registry searches was 2 (range 0-49). For six reviews, authors reported finding no relevant ongoing or completed studies as a result of their registry searches. Seven manuscripts provided the specific terms used to perform the registry search.

Cohen's kappa coefficient for inter-rater reliability with respect to whether systematic reviews included registry searches was 0.98, indicating excellent agreement.

\section{Discussion}

Clinical trials registries were not routinely included as part of the search strategies utilized for this group of systematic reviews published in high-impact general medical journals. Our results show that just 35\% of these systematic reviews reportedly incorporated searches from any clinical trials registry. When review authors did search trials registries, however, completed but unpublished studies were identified in approximately half of cases and ongoing studies identified in the majority of cases. These findings suggest that trials registries are an underutilized resource among investigators conducting systematic reviews.

Prior investigations have demonstrated that a substantial proportion of clinical trials are completed but never

Table 1 Characteristics of systematic reviews published in six major medical journals from July 2012 through June 2013

\begin{tabular}{|c|c|c|c|}
\hline Review characteristics & $\begin{array}{l}\text { All interventional reviews } \\
(n=117)\end{array}$ & $\begin{array}{l}\text { Searched a registry } \\
(n=41)\end{array}$ & $\begin{array}{l}\text { No registry search described } \\
(n=76)\end{array}$ \\
\hline \multicolumn{4}{|l|}{ Journal, $n(\%)$} \\
\hline Annals of Internal Medicine & 41 & $16(39)$ & $25(61)$ \\
\hline BMJ & 37 & $13(35)$ & $24(65)$ \\
\hline The Journal of the American Medical Association & 11 & $4(36)$ & $7(64)$ \\
\hline The Lancet & 12 & $4(33)$ & $8(67)$ \\
\hline The New England Journal of Medicine & 1 & $0(0)$ & $1(100)$ \\
\hline PLOS Medicine & 15 & $4(27)$ & $11(73)$ \\
\hline \multicolumn{4}{|l|}{ Funding source, $n(\%)^{a}$} \\
\hline Industry & 38 & $17(45)$ & $21(55)$ \\
\hline $\mathrm{NIH} /$ government & 81 & $29(36)$ & $52(64)$ \\
\hline Other & 32 & $11(34)$ & $21(66)$ \\
\hline None & 13 & $4(31)$ & $9(69)$ \\
\hline Manuscript reported PRISMA compliance & 38 & $18(47)$ & $20(53)$ \\
\hline Number of individual studies included in review, median (range) & $30(5-639)$ & $35(5-639)$ & $29(5-379)$ \\
\hline
\end{tabular}

${ }^{\mathrm{a}}$ Reviews with multiple funding sources are listed within all relevant categories; totals therefore add to more than $100 \%$.

${ }^{\mathrm{b}}$ National Institutes of Health. 


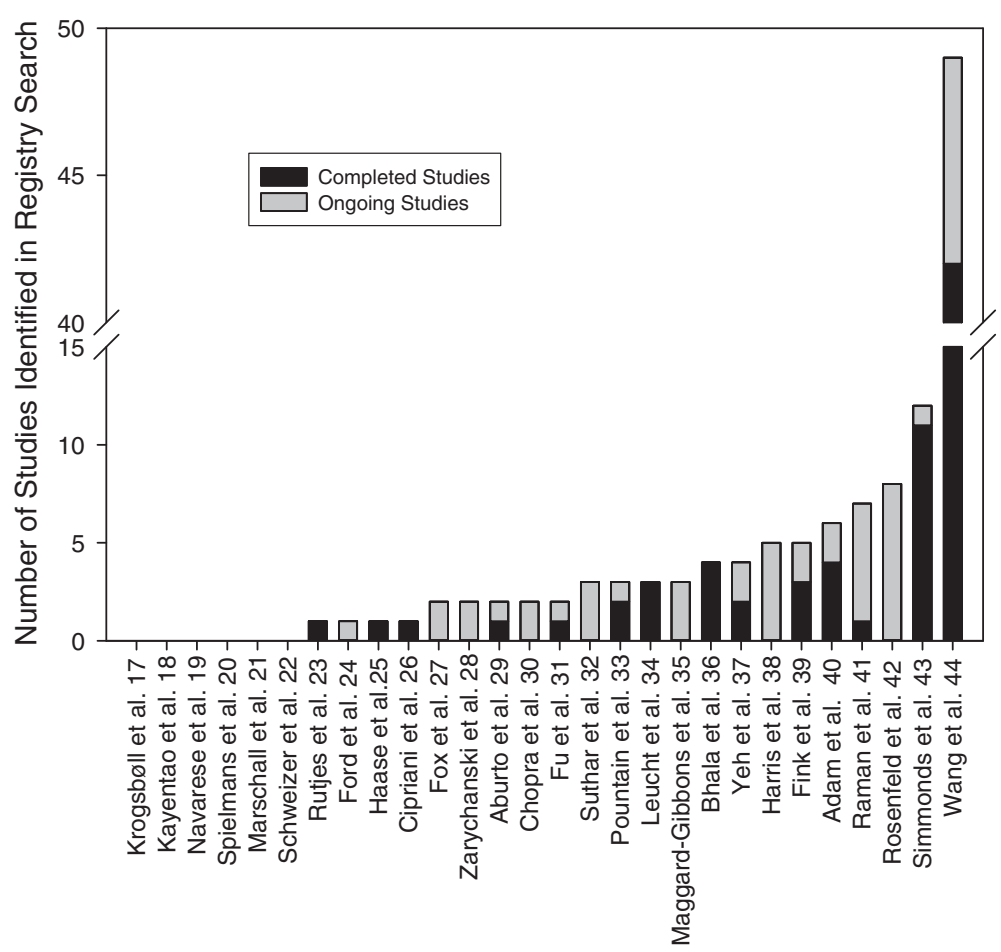

Figure 1 Registry search results by systematic review [17-44]. N =28; one review reported finding "several" relevant ongoing trials.

published [45]. Among trials registered with ClinicalTrials. gov, approximately one in three remain unpublished [46,47], and the rate of non-publication is almost as high for large trials [4]. These estimated rates of non-publication are particularly important given the increasing numbers of trials being registered and conducted. In January of 2005, 12,000 studies had been registered at ClinicalTrials.gov; by October 2013 over 152,000 studies had been registered [48]. Other registries have reported similar growth rates [49]. Thus, clinical trials registries potentially contain a large amount of trial information which is not available in the published literature.

In an effort to make ClinicalTrials.gov more effective at combating publication bias, the United States FDAAA of 2007 expanded the scope of ClinicalTrials.gov via the creation of a results database. The FDAAA mandated that results from nearly all phase II-IV human-subjects trials of FDA-approved drugs or devices conducted within the United States be submitted to ClinicalTrials.gov within one year of the study completion date, regardless of publication status [50]. As utilization of the results database improves, ClinicalTrials.gov will contain outcome data from an increasing number of otherwise unpublished studies. The availability of results will allow a trials registry search to not only inform investigators about the potential for publication bias but also allow for correction of this bias.

Even among registered studies without results available on ClinicalTrials.gov, registry entries are likely to contain information of significant value to the authors of systematic reviews. First, comparing registered studies to published studies allows identification of unpublished trials, and authors of systematic reviews can use the number and sample sizes of unpublished studies in combination with other methods such as funnel plots to better describe the possible impact of publication bias on effect estimates. For example, particular caution should be exercised when drawing conclusions from pooled data when registry searches reveal the existence of unpublished trials with large sample sizes relative to the subset of published trials. Second, even when trial results are published, examining registry entries can help review authors identify outcome reporting bias by allowing them to compare a priori planned outcomes to reported outcomes [51,52]. Additionally, registry entries frequently include contact information for study investigators, thereby providing review authors with the opportunity to contact investigators to request information about unpublished or incompletely reported studies. A recent comparison between published trial results and results posted on ClinicalTrials.gov also showed that ClinicalTrials.gov was far more likely than a published manuscript to include a complete accounting of adverse study events [53]. Finally, registry searches may reveal the existence of ongoing or recently completed trials, allowing the authors of systematic reviews to more accurately discuss their results within the context of ongoing research. 
Our results are similar to findings from an analysis of Cochrane systematic reviews published between 2008 and 2010 , in which trials registry searches were only included in $38 \%$ of reviews [54]. This study found that for the reviews in which registry search results were reported, relevant completed or ongoing trials were identified just $45 \%$ of the time. In our study, at least one relevant completed or ongoing trial was identified by $79 \%$ of the reviews reporting registry search results. One possible explanation for this difference is that the utility of registry searches may be increasing with time as trial pre-registration becomes more commonplace.

Since 2009 the Centre for Reviews and Dissemination has identified ClinicalTrials.gov and other public trials registries as important resources for identifying trial results [55]. Other commonly referenced guidelines for the conduct of systematic reviews, including those produced by the Institute of Medicine and the Agency for Healthcare Research \& Quality, have strongly recommended the use of a clinical trials registry search as part of a comprehensive search strategy since 2011 [12,13]. Further, the Cochrane Handbook recommends that Cochrane Review authors search registries in order to assess both for completed but unpublished and for ongoing trials [56]. Despite these recommendations, our results show that trials registries are not routinely utilized by systematic review authors. The PRISMA Statement, which has been endorsed by the World Association of Medical Editors, the Cochrane Collaboration, and numerous other organizations and journals, and provides a guide to the reporting of systematic reviews, does not explicitly advise authors to search trial registries [1]. While PRISMA is intended to be a guide to systematic review reporting, it is probably also used by investigators as a guide to systematic review conduct. As compliance with trial registration requirements and use of the ClinicalTrials.gov database for reporting results increase, revisions to PRISMA that place greater emphasis on incorporating registry data into systematic reviews may be useful in raising awareness among authors of systematic reviews of the potential importance of this resource.

Several limitations should be considered when interpreting these results. Not all systematic reviews address topics which can be studied with a clinical trial, and it is possible that even among the group of reviews which assessed interventions, some review authors decided not to search a trial registry because they thought that there were no relevant trials on the subject. However, registry data may be valuable even for authors conducting systematic reviews of subjects not amenable to clinical trials. Currently over 27,000 observational studies are registered with ClinicalTrials.gov, accounting for $18 \%$ of the database's registry entries, and the authors of observational studies are increasingly being encouraged to prospectively register their studies [48,57]. ClinicalTrials.gov functions as a significant source of registry data from noninterventional studies, and even systematic reviews that examine questions not easily tested with a clinical trial may benefit from registry searches. Additionally, we did not assess the impact of searching industry-sponsored results databases in this analysis, though these databases can also be important sources of unpublished trial data [58].

The external validity of our results may also be limited. We studied systematic reviews published in a group of high-impact general medicine journals; it is possible that different patterns would be observed among other journals. Also, it is probable that some systematic reviews meeting our inclusion criteria were missed by our search strategy. However, the strategy we employed has previously been validated as a sensitive method of identifying systematic reviews, and it is unlikely that any systematic reviews we overlooked would have a substantively different rate of searching trials registries.

\section{Conclusions}

In this sample of recently published systematic reviews in major medical journals, searches of clinical trials registries were not routinely utilized. In the cases where registry searches were performed, authors identified relevant completed or ongoing trials more than three quarters of the time. More consistent use of trials registry databases may improve the identification of publication and outcome reporting biases and increase the validity of estimated effects of medical treatments in systematic reviews.

\section{Additional file}

Additional file 1: Systematic reviews. Records published between July 1, 2012 and June 30, 2013 and identified via PubMed search.

\section{Abbreviations}

ICMJE: International Committee of Medical Journal Editors; FDAAA: Food and Drug Administration Amendments Act; JAMA: The Journal of the American Medical Association; WHO: World Health Organization; PRISMA: Preferred Reporting Items for Systematic Reviews and Meta-Analyses; ICTRP: International Clinical Trials Registry Platform.

\section{Competing interests}

The authors declare that they have no competing interests.

\section{Authors' contributions}

All authors contributed to the study concept and design. Data acquisition was performed by CWJ and LGK. Data analysis and interpretation was performed by CWJ, LGK, MAW, and TPM. MAW provided statistical expertise. The initial manuscript was drafted by CWJ, LGK, and TPM; all authors contributed to subsequent revisions. CWJ had full access to all the data in the study and had final responsibility for the decision to submit for publication. All authors read and approved the final manuscript.

\section{Authors' information}

CWJ is an investigator on a study sponsored by Roche Diagnostics which provides financial support to his department. MAW is supported by the National Center for Research Resources and the National Center for Advancing Translational Sciences through grant UL1 TR001111-01, and TPM 
is supported by the National Institute on Aging through grant K23 AG038548. These sponsors had no role in the study design; collection, analysis, and interpretation of the data; writing of the report; or in the decision to submit the manuscript for publication.

\section{Author details}

${ }^{1}$ Department of Emergency Medicine, Cooper Medical School of Rowan University, One Cooper Plaza, Suite 152, Camden, NJ 08103, USA. ${ }^{2}$ Department of Emergency Medicine, University of North Carolina Chapel Hill, Chapel Hill, USA. ${ }^{3}$ Departments of Internal Medicine and Biostatistics, University of North Carolina Chapel Hill, Chapel Hill, USA.

Received: 25 July 2014 Accepted: 29 September 2014

Published: 27 October 2014

\section{References}

1. Liberati A, Altman DG, Tetzlaff J, Mulrow C, Gotzsche PC, loannidis JP, Clarke M, Devereaux PJ, Kleijnen J, Moher D: The PRISMA statement for reporting systematic reviews and meta-analyses of studies that evaluate health care interventions: explanation and elaboration. J Clin Epidemiol 2009, 62(10):e1-e34.

2. Spoor P, Airey M, Bennett C, Greensill J, Williams R: Use of the capture-recapture technique to evaluate the completeness of systematic literature searches. BMJ 1996, 313:342-343.

3. Peters JL, Sutton AJ, Jones DR, Abrams KR, Rushton L: Comparison of two methods to detect publication bias in meta-analysis. JAMA 2006, 295:676-680.

4. Jones CW, Handler L, Crowell KE, Keil LG, Weaver MA, Platts-Mills TF: Non-publication of large randomized clinical trials: cross sectional analysis. BMJ 2013, 347:f6104

5. Lau J, loannidis JP, Terrin N, Schmid CH, Olkin I: The case of the misleading funnel plot. BMJ 2006, 333:597-600

6. Terrin N, Schmid CH, Lau J: In an empirical evaluation of the funnel plot, researchers could not visually identify publication bias. J Clin Epidemiol 2005, 58:894-901.

7. De Angelis C, Drazen JM, Frizelle FA, Haug C, Hoey J, Horton R, Kotzin S, Laine C, Marusic A, Overbeke AJ, Schroeder TV, Sox HC, Van Der Weyden MB: Clinical trial registration: a statement from the International Committee of Medical Journal Editors. N Engl J Med 2004, 351(12):1250-1251.

8. Food and Drug Administration Amendments Act of 2007. US Public Law 110-85. (2007, Sept 27); 21 USC 301.

9. Simes RJ: Publication bias: the case for an international registry of clinical trials. J Clin Oncol 1986, 4:1529-1541.

10. Tonks A: A clinical trials register for Europe. BMJ 2002, 325:1314-1315.

11. Jefferson T, Jones MA, Doshi P, Del Mar CB, Heneghan CJ, Hama R, Thompson MJ, Spencer EA, Onakpoya I, Mahtani KR, Nunan D, Howick J, Heneghan CJ: Neuraminidase inhibitors for preventing and treating influenza in healthy adults and children. Cochrane Database Syst Rev 2012, 1:CD008965.

12. Institute of Medicine: Finding what works in health care: standards for systematic reviews. In 2011. [www.iom.edu/Reports/2011/Finding-WhatWorks-in-Health-Care-Standards-for-Systematic-Reviews/Standards.aspx]. Accessed April, 2014

13. Relevo R, Balshem H: Finding evidence for comparing medical interventions: AHRQ and the Effective Health Care Program. J Clin Epidemiol 2011, 64(11):1168-1177.

14. Montori VM, Wilczynski NL, Morgan D, Haynes RB: Optimal search strategies for retrieving systematic reviews from Medline: analytical survey. BMJ 2005, 330:68.

15. International Clinical Trials Registry Platform, WHO Registry Network. [http://www.who.int/ictrp/network/en/]. Accessed 02/11/2014.

16. Myung SK, Ju W, Cho B, Oh SW, Park SM, Koo BK, Park BJ: Efficacy of vitamin and antioxidant supplements in prevention of cardiovascular disease: systematic review and meta-analysis of randomised controlled trials. BMJ 2013, 346:f10.

17. Krogsboll LT, Jorgensen KJ, Gronhoj Larsen C, Gotzsche PC: General health checks in adults for reducing morbidity and mortality from disease: cochrane systematic review and meta-analysis. BMJ 2012, 345:e7191.

18. Kayentao K, Garner P, van Eijk AM, Naidoo I, Roper C, Mulokozi A, MacArthur JR, Luntamo M, Ashorn P, Doumbo OK, ter Kuile FO: Intermittent preventive therapy for malaria during pregnancy using 2 vs 3 or more doses of sulfadoxine-pyrimethamine and risk of low birth weight in Africa: systematic review and meta-analysis. JAMA 2013, 309(6):594-604
19. Navarese EP, Gurbel PA, Andreotti F, Tantry U, Jeong $Y H$, Kozinski M, Engstrom T, Di Pasquale G, Kochman W, Ardissino D, Kedhi E, Stone GW Kubica J: Optimal timing of coronary invasive strategy in non-STsegment elevation acute coronary syndromes: a systematic review and meta-analysis. Ann Intern Med 2013, 158(4):261-270.

20. Spielmans Gl, Berman MI, Linardatos E, Rosenlicht NZ, Perry A, Tsai AC: Adjunctive atypical antipsychotic treatment for major depressive disorder: a meta-analysis of depression, quality of life, and safety outcomes. PLoS Med 2013, 10:e1001403.

21. Marschall J, Carpenter CR, Fowler S, Trautner BW: Antibiotic prophylaxis for urinary tract infections after removal of urinary catheter: meta-analysis. BMJ 2013, 346:f3147.

22. Schweizer M, Perencevich E, McDanel J, Carson J, Formanek M, Hafner J, Braun B, Herwaldt $L$ : Effectiveness of a bundled intervention of decolonization and prophylaxis to decrease Gram positive surgical site infections after cardiac or orthopedic surgery: systematic review and meta-analysis. BMJ 2013, 346:f2743.

23. Rutjes AW, Juni P, da Costa BR, Trelle S, Nuesch E, Reichenbach S: Viscosupplementation for osteoarthritis of the knee: a systematic review and meta-analysis. Ann Intern Med 2012, 157:180-191.

24. Ford JA, Elders A, Shyangdan D, Royle P, Waugh N: The relative clinical effectiveness of ranibizumab and bevacizumab in diabetic macular oedema: an indirect comparison in a systematic review. BMJ 2012, 345:e5182.

25. Haase N, Perner A, Hennings LI, Siegemund M, Lauridsen B, Wetterslev M, Wettersley J: Hydroxyethyl starch $130 / 0.38-0.45$ versus crystalloid or albumin in patients with sepsis: systematic review with meta-analysis and trial sequential analysis. BMJ 2013, 346:f839.

26. Cipriani A, Hawton K, Stockton S, Geddes JR: Lithium in the prevention of suicide in mood disorders: updated systematic review and meta-analysis. BMJ 2013, 346:f3646

27. Fox BD, Kahn SR, Langleben D, Eisenberg MJ, Shimony A: Efficacy and safety of novel oral anticoagulants for treatment of acute venous thromboembolism: direct and adjusted indirect meta-analysis of randomised controlled trials. BMJ 2012, 345:e7498.

28. Zarychanski R, Abou-Setta AM, Turgeon AF, Houston BL, Mclntyre L, Marshall JC, Gergusson DA: Association of hydroxyethyl starch administration with mortality and acute kidney injury in critically ill patients requiring volume resuscitation: a systematic review and meta-analysis. JAMA 2013, 309(7):678-688.

29. Aburto NJ, Ziolkovska A, Hooper L, Elliott P, Cappuccio FP, Meerpohl JJ: Effect of lower sodium intake on health: systematic review and meta-analyses. BMJ 2013, 346:f1326.

30. Chopra V, Anand S, Hickner A, Buist M, Rogers MA, Saint S, Flanders SA: Risk of venous thromboembolism associated with peripherally inserted central catheters: a systematic review and meta-analysis. Lancet 2013, 382(9889):311-325.

31. Fu R, Selph S, McDonagh M, Peterson K, Tiwari A, Chou R, Helfand M: Effectiveness and harms of recombinant human bone morphogenetic protein-2 in spine fusion: a systematic review and meta-analysis. Ann Intern Med 2013, 158(12):890-902.

32. Suthar AB, Lawn SD, del Amo J, Getahun H, Dye C, Sculier D, Sterling TR, Chaisson RE, Williams BG, Harries AD, Granich RM: Antiretroviral therapy for prevention of tuberculosis in adults with HIV: a systematic review and meta-analysis. PLoS Med 2012, 9:e1001270.

33. Pountain SJ, Roffe C: Does routine oxygen supplementation in patients with acute stroke improve outcome? BMJ 2012, 345:e6976.

34. Leucht S, Cipriani A, Spineli L, Mavridis D, Orey D, Richter F, Samara M, Barbui C, Engel RR, Geddes JR, Kissling W, Stapf MP, Lassig B, Salanti G, Davis JM: Comparative efficacy and tolerability of 15 antipsychotic drugs in schizophrenia: a multiple-treatments meta-analysis. Lancet 2013, 382(9896):951-962.

35. Maggard-Gibbons M, Maglione M, Livhits M, Ewing B, Maher AR, Hu J, Li Z, Shekelle PG: Bariatric surgery for weight loss and glycemic control in nonmorbidly obese adults with diabetes: a systematic review. JAMA 2013, 309(21):2250-2261.

36. Bhala N, Emberson J, Merhi A, Abramson S, Arber N, Baron JA, Bombardier C, Cannon C, Farkouh ME, FitzGerald GA, Goss P, Halls H, Hawk E, Hawkey C, Hennekens C, Hochberg M, Holland LE, Kearney PM, Laine L, Lanas A, Lance P, Laupacis A, Oates J, Patrono C, Schnitzer TJ, Solomon S, Tugwell P, Wilson K, Wittes J, Baigent C, et al: Vascular and upper gastrointestinal effects of non-steroidal anti-inflammatory drugs: meta-analyses of individual participant data from randomised trials. Lancet 2013, 382(9894):769-779.

37. Yeh HC, Brown TT, Maruthur N, Ranasinghe P, Berger Z, Suh YD, Wilson LM, Haberl EB, Brick J, Bass EB, Golden SH: Comparative effectiveness and safety of methods of insulin delivery and glucose monitoring for diabetes mellitus: a systematic review and meta-analysis. Ann Intern Med 2012, 157(5):336-347. 
38. Harris T, Thomas GO, Brohi K: Early fluid resuscitation in severe trauma. BMJ 2012, 345:e5752.

39. Fink HA, Wilt TJ, Eidman KE, Garimella PS, MacDonald R, Rutks IR, Brasure M, Kane RL, Ouellette J, Monga M: Medical management to prevent recurrent nephrolithiasis in adults: a systematic review for an American College of Physicians Clinical Guideline. Ann Intern Med 2013, 158(7):535-543.

40. Adam SS, McDuffie JR, Ortel TL, Williams JW Jr: Comparative effectiveness of warfarin and new oral anticoagulants for the management of atrial fibrillation and venous thromboembolism: a systematic review. Ann Intern Med 2012, 157:796-807.

41. Raman G, Moorthy D, Hadar N, Dahabreh IJ, O'Donnell TF, Thaler DE, Feldmann E, Lau J, Kitsios GD: Management strategies for asymptomatic carotid stenosis: a systematic review and meta-analysis. Ann Intern Med 2013, 158(9):676-685.

42. Rosenfeld JV, Maas Al, Bragge P, Morganti-Kossmann MC, Manley GT, Gruen RL: Early management of severe traumatic brain injury. Lancet 2012, 380:1088-1098.

43. Simmonds MC, Brown JV, Heirs MK, Higgins JP, Mannion RJ, Rodgers MA Stewart LA: Safety and effectiveness of recombinant human bone morphogenetic protein-2 for spinal fusion: a meta-analysis of individualparticipant data. Ann Intern Med 2013, 158(12):877-889.

44. Wang SY, Olson-Kellogg B, Shamliyan TA, Choi JY, Ramakrishnan R, Kane RL: Physical therapy interventions for knee pain secondary to osteoarthritis: a systematic review. Ann Intern Med 2012, 157:632-644.

45. Turner EH, Knoepflmacher D, Shapley L: Publication bias in antipsychotic trials: an analysis of efficacy comparing the published literature to the US Food and Drug Administration database. PLoS Med 2012, 9:e1001189.

46. Ross JS, Mulvey GK, Hines EM, Nissen SE, Krumholz HM: Trial publication after registration in ClinicalTrials.gov: a cross-sectional analysis. PLOS Med 2009, 6:e1000144.

47. Ross JS, Tse T, Zarin DA, Xu H, Zhou L, Krumholz HM: Publication of NIH funded trials registered in ClinicalTrials.gov: cross sectional analysis. BMJ 2011, 344:d7292.

48. ClinicalTrials.gov. [http://clinicaltrials.gov/ct2/resources/trends\#Registered StudiesOverTime]. Accessed 09/01/2014.

49. Current Controlled Trials. [http://www.controlled-trials.com/]. Accessed $02 /$ $11 / 2014$

50. ClinicalTrials.gov FDAAA 801 Requirements. [http://clinicaltrials.gov/ct2/ manage-recs/fdaaa]. Accessed 02/11/2014.

51. Chan AW, Hrobjartsson A, Haahr MT, Gotzsche PC, Altman DG: Empirical evidence for selective reporting of outcomes in randomized trials: comparison of protocols to published articles. JAMA 2004, 291:2457-2465.

52. Dwan K, Altman DG, Arnaiz JA, Bloom J, Chan AW, Cronin E, Decullier E, Easterbrook PJ, Von Elm E, Gamble C, Ghersi D, loannidis JP, Simes J, Williamson PR: Systematic review of the empirical evidence of study publication bias and outcome reporting bias. PLOS One 2008, 3(8):e3081.

53. Riveros C, Dechartres A, Perrodeau E, Haneef R, Boutron I, Ravaud P. Timing and completeness of trial results posted at ClinicalTrials.gov and published in journals. PLoS Med 2013, 10(12):e1001566.

54. van Enst WA, Scholten RJ, Hooft L: Identification of additional trials in prospective trial registers for Cochrane systematic reviews. PLoS One 2012, 7(8):e42812.

55. Centre for Reviews and Dissemination, University of York: Systematic Reviews: CRD's guidance for undertaking reviews in health care. York: University of York; 2009

56. Higgins JPT, Green S: Cochrane handbook for systematic reviews of interventions. version 5.1.0. Cochrane Collaboration 2011, www.cochranehandbook.org.

57. Should protocols for observational research be registered? Lancet 2010 375:348.

58. Potthast R, Vervölgyi V, McGauran N, Kerekes MF, Wieseler B, Kaiser T: Impact of inclusion of industry trial results registries as an information source for systematic reviews. PLoS One 2014, 9(4):e92067.

doi:10.1186/2046-4053-3-126

Cite this article as: Jones et al: Clinical trials registries are under-utilized in the conduct of systematic reviews: a cross-sectional analysis. Systematic Reviews 2014 3:126.

\section{Submit your next manuscript to BioMed Central and take full advantage of:}

- Convenient online submission

- Thorough peer review

- No space constraints or color figure charges

- Immediate publication on acceptance

- Inclusion in PubMed, CAS, Scopus and Google Scholar

- Research which is freely available for redistribution
C Biomed Central 